OPEN ACCESS

Edited by:

Cintia Roodveldt,

Andalusian Molecular Biology and

Regenerative Medicine Centre

(CABIMER) - CSIC, Spain

Reviewed by:

Janice Eva Arlee Braun,

University of Calgary, Canada

Konrad Ernst Zinsmaier,

University of Arizona, USA

*Correspondence:

Lucia Tabares

Itabares@us.es

${ }^{\dagger}$ Present Address:

Elena Lopez-Ortega,

Solomon H. Snyder Department of Neuroscience, Johns Hopkins University School of Medicine,

Baltimore, MD, USA

Specialty section

This article was submitted to

Neurodegeneration,

a section of the journal

Frontiers in Neuroscience

Received: 18 December 2016

Accepted: 19 January 2017

Published: 10 February 2017

Citation:

Lopez-Ortega E, Ruiz R and Tabares L

(2017) CSP $\alpha$, a Molecular

Co-chaperone Essential for Short and Long-Term Synaptic Maintenance.

Front. Neurosci. 11:39

doi: 10.3389/fnins.2017.00039

\section{CSP $\alpha$, a Molecular Co-chaperone Essential for Short and Long-Term Synaptic Maintenance}

\author{
Elena Lopez-Ortega ${ }^{1 \dagger}$, Rocío Ruiz ${ }^{2}$ and Lucia Tabares ${ }^{1 *}$
}

${ }^{1}$ Department of Medical Physiology and Biophysics, School of Medicine, University of Seville, Seville, Spain, ${ }^{2}$ Department of Biochemistry and Molecular Biology, School of Pharmacy, University of Seville, Seville, Spain

Cysteine string protein $\alpha(\mathrm{CSP} \alpha)$ is a vesicle protein located in the presynaptic terminal of most synapses. CSP $\alpha$ is an essential molecular co-chaperone that facilitates the correct folding of proteins and the assembly of the exocytic machinery. The absence of this protein leads to altered neurotransmitter release and neurodegeneration in multiple model systems, from flies to mice. In humans, CSP $\alpha$ mutations are associated with the development of neuronal ceroid lipofuscinosis ( $\mathrm{NCL}$ ), a neurodegenerative disease characterized by intracellular accumulation of lysosomal material. Here, we review the physiological role of CSP $\alpha$ and the pathology resulting from the homozygous deletion of the gene or its mutations. In addition, we investigate whether long-term moderate reduction of the protein produces motor dysfunction. We found that 1-year-old CSP $\alpha$ heterozygous mice display a reduced ability to sustain motor unit recruitment during repetitive stimulation, which indicates that physiological levels of CSP $\alpha$ are required for normal neuromuscular responses in mice and, likely, in humans.

Keywords: cysteine string protein, co-chaperone, motor neurons, synaptic transmission, neuromuscular junction, $\operatorname{cSP} \alpha$

\section{PROTEIN DESCRIPTION}

Cysteine string protein $\alpha(\mathrm{CSP} \alpha)$ (Dnajc5) is a highly conserved protein (Figure 1A) typically associated with the membrane of synaptic vesicles and secretory granules (Zinsmaier et al., 1990). It contains a DNA-J domain characteristic of Hsp40 co-chaperones. This domain interacts with the $70 \mathrm{kDa}$ heat shock cognate protein (Hsc70) (Braun et al., 1996) and regulates the refolding of client proteins (Hennessy et al., 2005). A linker region connects the DNA-J domain with the cysteine string domain. The cysteine string domain is approximately 25 -amino-acids long and contains 13-15 cysteines, most of them palmitoylated. Palmitoylation is essential to target CSP $\alpha$ to synaptic vesicles and to promote neurotransmitter release (Arnold et al., 2004). CSP $\alpha$ also contains a C-terminal domain, the function of which is not well-understood. 


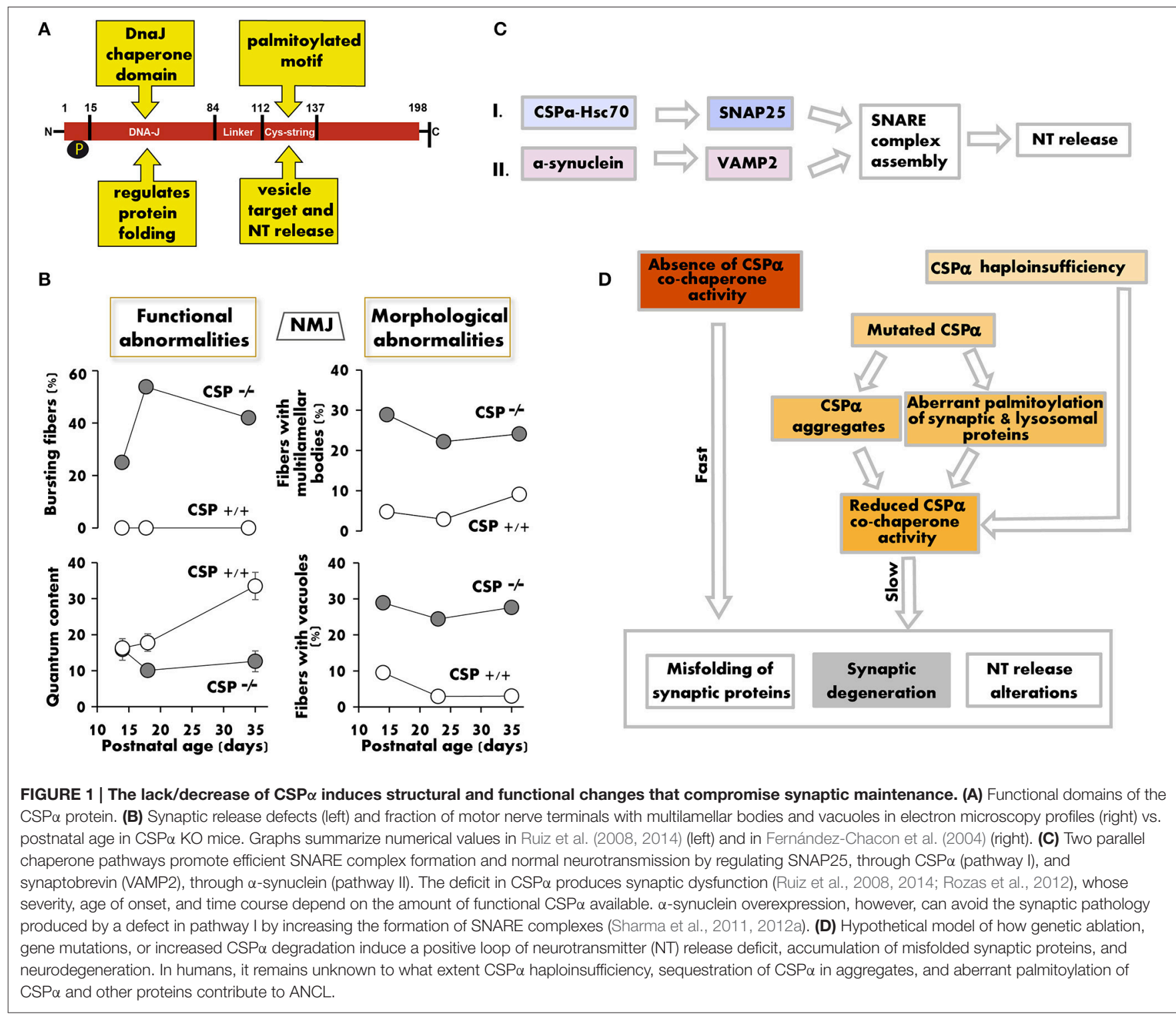

\section{CSP $\alpha$ DEFICIENCY AND SYNAPTIC DYSFUNCTION}

$\operatorname{CSP} \alpha$ is not essential for synaptogenesis, but it is required for normal neurotransmission and neuronal maintenance in flies (Zinsmaier et al., 1994), worms (Kashyap et al., 2014), mice (Fernández-Chacon et al., 2004), and humans (Nosková et al., 2011). Deletion of the $\operatorname{CSP} \alpha$ gene in Drosophila produces an embryonic semilethal phenotype, and flies that survive to adulthood present neurotransmitter release alterations and temperature-sensitive paralysis. Synaptic defects in CSP $\alpha$-null $(\mathrm{CSP} \alpha \mathrm{KO})$ mice start early after birth, and death occurs before 3 months of age. Both motor and sensory neurons are affected by the lack of $\operatorname{CSP} \alpha$ (Fernández-Chacon et al., 2004; Schmitz et al., 2006). In motor nerve terminals, the first sign of functional alteration appears at 2 weeks of age and consists of repeated bursts of high-frequency spontaneous release (Ruiz et al., 2008) (Figure 1B). Only 4 days later, the terminal displays multiple functional alterations such as reduced quantum content, low release probability, increased short-term facilitation during repetitive stimulation, and reduced calcium sensitivity of the secretory machinery (Ruiz et al., 2008, 2014). In addition, the size of the readily releasable pool of synaptic vesicles is decreased (Rozas et al., 2012; Ruiz et al., 2014).

\section{CSP $\alpha$ AS A MOLECULAR CO-CHAPERONE}

$\operatorname{CSP} \alpha$ interacts with several proteins that participate in exo/endocytosis, including syntaxin, synaptotagmin, $\mathrm{N}$ - and P/Qtype calcium channels, and dynamin 1 (for a review see Burgoyne and Morgan, 2015). The functional significance of many of these interactions is not well established. However, 
one of the best-known functions of $\operatorname{CSP} \alpha$ is its role as a cochaperone (Chamberlain and Burgoyne, 2000; Zinsmaier, 2010; Donnelier and Braun, 2014). CSP $\alpha$ interacts with Hsc70 and together refold client proteins such as SNAP25 (Sharma et al., 2011, 2012a; Zhang et al., 2012). SNAP25 is required for the assembly of the SNARE (soluble N-ethylmaleimide-sensitive factor attachment receptor) complex, formed by synaptobrevin, syntaxin, and SNAP25, which in turn is essential for exocytosis and neurotransmitter release (Figure 1C). In $\mathrm{CSP} \alpha \mathrm{KO}$ mice, both SNAP25 and SNARE complex levels are reduced by half (Chandra et al., 2005).

\section{CSP $\alpha$ AND NEURODEGENERATION}

In $\operatorname{CSP} \alpha \mathrm{KO}$ motor nerve terminals, hallmarks of degeneration (i.e., vacuoles and multilamellar bodies) appear very early (Fernández-Chacon et al., 2004), even before the evoked release defects become apparent (Figure 1B, left graphs). The degeneration is more prominent in cells with high electrical activity, such as motor neurons, photoreceptors, and GABAergic neurons (Fernández-Chacon et al., 2004; Schmitz et al., 2006; García-Junco-Clemente et al., 2010), suggesting that high synaptic activity potentiates degeneration, which in turn may increase the release deficit (Figure 1D).

At the molecular level, it has been proposed that SNAP25 reduction plays a major role in neurodegeneration. This hypothesis is reinforced by the fact that SNAP25 overexpression in $\operatorname{CSP} \alpha \mathrm{KO}$ mice prevents neurodegeneration (Sharma et al., 2012a). Nevertheless, SNAP25 heterozygous mice, with 50\% of the protein, are phenotypically normal and do not develop neurodegeneration (Washbourne et al., 2002), indicating that solely reducing functional SNAP25 is not sufficient to produce the pathology. On the other hand, misfolded SNAP25 could have a dominant negative effect over the normally folded protein copies, or ubiquitinated SNAP25 molecules could accumulate in the proteasome, interfering with its normal function. Surprisingly, however, the fact that pharmacological inhibition of the proteasome increases SNAP25 and SNARE complex levels, and, hence, improves synaptic function in CSP $\alpha$ depleted cells (Sharma et al., 2012b), has challenged this last hypothesis.

Remarkably, neurodegeneration in $\operatorname{CSP} \alpha \mathrm{KO}$ mice is prevented when SNARE complexes are increased by the overexpression of $\alpha$-synuclein (Sharma et al., 2011, 2012a), in spite of the fact that SNAP25 levels are not restored and, presumably, the amount of misfolded SNAP25 is not reduced. Overexpression of the mutated form of $\alpha$-synuclein A53T in $\operatorname{CSP} \alpha \mathrm{KO}$ mice restores life span and motor function as well as wild-type (WT) $\alpha$-synuclein (Chandra et al., 2005). On the other hand, overexpression of A30P $\alpha$-synuclein does not rescue survival, but can transitorily ameliorate the release deficit and the calcium sensitivity defect in motor nerve terminals of CSP $\alpha \mathrm{KO}$ mice (Ruiz et al., 2014). The partial effect of A30P $\alpha$-synuclein is likely due to its limited ability to increase the formation of SNARE complexes. These findings suggest that two parallel pathways regulate SNARE complex formation (Figure 1C), and raise the question of how the neurodegeneration program is activated in the absence of $\operatorname{CSP} \alpha$. The molecular basis of the neurodegeneration is unknown, but a possibility is that neurodegeneration is linked to the reduced ability of the synapse to form SNARE complexes and, therefore, to the mismatch between functional demands and efficient release.

\section{CSP $\alpha$ DEFICIENCY IN HUMANS}

Neuronal Ceroid Lipofuscinosis (NCLs) constitute a heterogeneous group of inherited neurodegenerative disorders characterized by lysosomal accumulation of autofluorescent ceroid-lipofuscin aggregates in neurons and other cell types. The clinical symptoms of NCLs include seizures, movement disorders, cognitive deterioration, and progressive dementia, followed by a premature death. The majority of NCL cases affect children, and only $10 \%$ of total cases are in adults.

In recent years, two mutations in the gene that encodes $\operatorname{CSP} \alpha, D N A J C 5$, have been linked to the development of adultonset NCL (ANCL) (MIM \#162350). These mutations consist of a point mutation (p.L115R) and an in-frame codon deletion (p.L116 $\Delta$ ), both affecting dileucine residues located in the cysteine string domain of $\operatorname{CSP} \alpha$ (Nosková et al., 2011). This domain is highly palmitoylated and mediates the membrane binding and intracellular targeting of $\operatorname{CSP} \alpha$. Therefore, mutations in this region may explain the diffuse intracellular localization of $\operatorname{CSP} \alpha$ observed in the neurons of ANCL patients. Moreover, the mutated forms of $\operatorname{CSP} \alpha$ present an increased tendency to self-associate, forming detergent-resistant aggregates. These aggregates interfere with $\mathrm{WT} \operatorname{CSP} \alpha$ proteins, reducing the cochaperone function of CSP $\alpha$ in neurons (Nosková et al., 2011; Greaves et al., 2012; Zhang and Chandra, 2014) (Figure 1D), which is likely one of the main reasons why the NCL-linked DNAJC5 gene mutations display an autosomal dominant (AD) inheritance pattern. Additionally, a dominant effect of mutated $\operatorname{CSP} \alpha$ on the palmitoylation pattern of lysosomal and synaptic proteins has been suggested as a mechanism for the development of DNAJC5-linked ANCL (Henderson et al., 2016).

\section{LONG-TERM MODERATE CSP $\alpha$ DEFICIENCY ALTERS MOTOR RESPONSES}

Interestingly, only homozygous $\mathrm{CSP} \alpha \mathrm{KO}$ mice present synaptic defects, while heterozygous mutant mice appear phenotypically normal up to 3 months of age (Fernández-Chacon et al., 2004). Given the late onset of AD-ANCL (around 30 years of age), we studied the motor function of 1-year-old $\operatorname{CSP} \alpha$ heterozygous mice. Motor performance was first assessed with Balance and Grip Strength tests (Figure 2A). The balance was measured by placing the mouse on a horizontal pole suspended in the air. The pole was rotated manually at a constant speed of one rotation cycle per second (Figure $2 \mathrm{~A}$ left). The grip strength test consisted of suspending the mouse from the pole by its forelimbs (Figure 2A right). In both trials, the amount of time the mouse remained suspended from the pole (maximum $10 \mathrm{~s}$ ) 
A
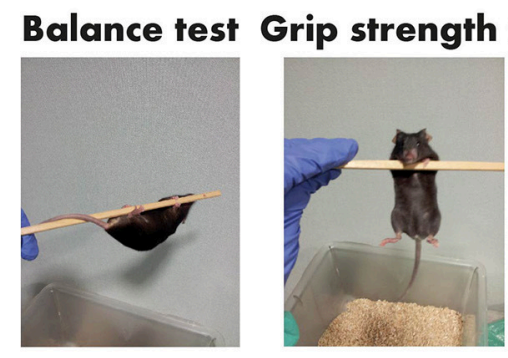

B

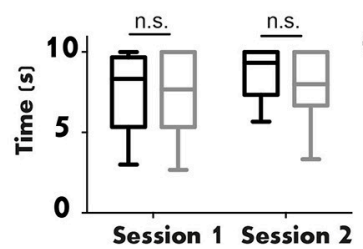

Grip strength

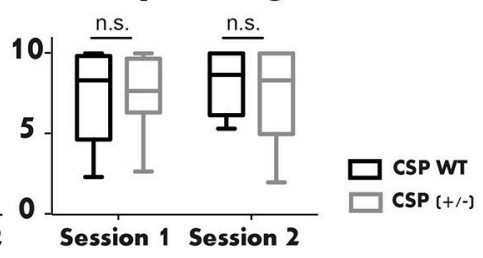

C

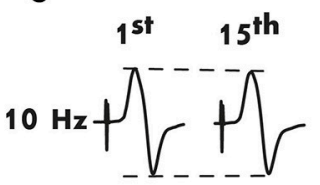

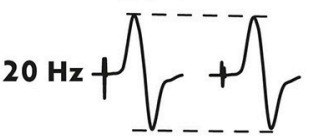

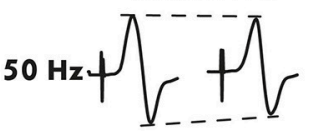

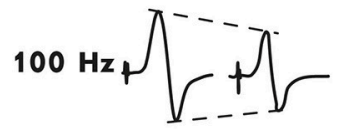

D

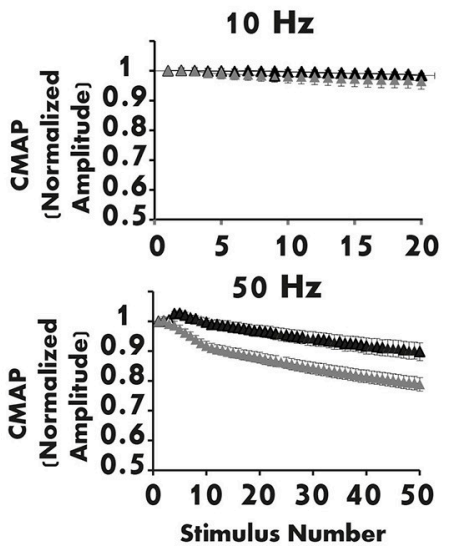

Depression

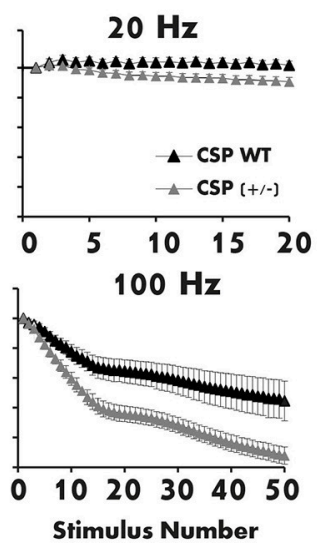

E

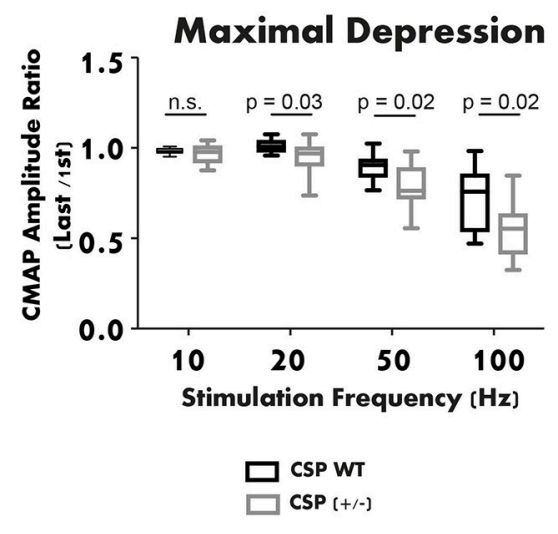

FIGURE 2 | The neuromuscular function of 1-year-old CSP $\alpha$ heterozygous mice is altered. (A,B) Balance and grip strength tests show no significant differences between CSP $\alpha$ heterozygous $(n=27)$ and WT mice $(n=9)$. The plot illustrates the average values obtained in three replicates/session for each genotype (Mann-Whitney $U$ test). (C) Representative recordings of CMAPs in a CSP $\alpha^{+/+}$mouse after supramaximal stimulation of the sciatic nerve (1st and 15th responses at different stimulation frequencies). (D) Depression of the neuromuscular responses (CMAP normalized amplitude) is significantly larger in CSP $\alpha^{+/-}(n=25)$ than in WT mice $(n=7)$ during stimulation trains at 20,50 , and $100 \mathrm{~Hz}$. (E) Maximal depression of the neuromuscular response (normalized) at different stimulation frequencies in CSP $\alpha^{+/-}(n=24-25)$ and WT mice $(n=7)$ (Mann-Whitney $U$ test). Either sex experimental mice (C57BL/6 background) were used. The mouse line was kindly donated by Dr. Südhof. All experiments were performed according to the guidelines of the European Council Directive for the Care of Laboratory Animals. The protocol was approved by the Ethics Committee for Animal Experimentation of the Junta de Andalucía (ref. 23-11-2015-364).

was measured. Two sessions separated by 1 week were performed for each test (Figure 2B). Data obtained from the neurological tests showed no significant differences between WT and $\operatorname{CSP} \alpha$ heterozygous mice, in either balance or grip strength.

Next, the neuromuscular response of $\operatorname{CSP} \alpha$ heterozygous mice was studied using electromyography (EMG). Evoked Compound Motor Action Potentials (CMAPs) were recorded from the right lateral gastrocnemius of anesthetized mice. Stimulation needle electrodes were placed at the sciatic notch and the head of the fibula (Ruiz et al., 2005). The active recording electrode was placed in the medial region of the recorded muscle. The reference electrode was inserted at the base of the fifth foot phalanx. The ground electrode was located at the base of the tail. Brief supramaximal stimulation pulses were applied at $10 \mathrm{~Hz}(2 \mathrm{~s})$, $20 \mathrm{~Hz}(1 \mathrm{~s}), 50 \mathrm{~Hz}(1 \mathrm{~s})$, and $100 \mathrm{~Hz}(0.5 \mathrm{~s})$. Representative recordings of CMAPs registered during the trains are shown in Figure 2C. The study revealed enhanced depression with stimulations between 20 and $100 \mathrm{~Hz}$ in CSP $\alpha$ heterozygous mice compared to WT littermates (Figure 2D). The mean maximal depression was $5 \%(20 \mathrm{~Hz}), 12 \%(50 \mathrm{~Hz})$, and $25 \%(100 \mathrm{~Hz})$ lower in $\operatorname{CSP} \alpha$ heterozygous than in WT mice, while no significant difference was observed at $10 \mathrm{~Hz}$ (Figure 2E). Remarkably, the depression in the EMG recordings was similar to that seen in 3-week-old CSP $\alpha$ KO mice (Fernández-Chacon et al., 2004), a phenotype not observed in $\operatorname{CSP} \alpha^{+/-}$mice up to 3 month of age. These results indicate that, over time, a moderate reduction of $\operatorname{CSP} \alpha$ expression alters the ability of the neuromuscular system to respond normally to stimulation.

\section{FUTURE DIRECTIONS}

The multiple functions of $\operatorname{CSP} \alpha$ range from acting as a chaperone, participating in the assembly and dissociation of multi-protein complexes, and regulating $\mathrm{Ca}^{2+}$ sensitivity for neurotransmitter release. The severe functional and structural changes that take place in the absence of $\operatorname{CSP} \alpha$ in invertebrate and vertebrate organism models confirm the importance of this protein in synapse maintenance and neurotransmitter release. In humans, $\operatorname{CSP} \alpha$ mutations are associated with the development of AD-ANCL, synaptic degeneration, and neuronal 
loss. Therefore, although both the reduction of CSP $\alpha$ expression and the presence of $\operatorname{CSP} \alpha$ mutations are pathogenic to the synapse, the severity and time course of the neurological impairments may vary from severe, including premature death, to mild, depending on the amount of functional CSP $\alpha$ in each case. The moderate decrease in CSP $\alpha$ and SNARE complexes in neurons over time could result in motor function impairment and, in addition, influence the evolution of common agerelated neurodegenerative disorders, such as Alzheimer's and Parkinson's diseases. Future challenges are to identify patients with reduced levels of molecular chaperones (such as CSP $\alpha$ ), decipher the mechanisms responsible for the molecular deficit, understand how the homeostasis of the synapse is altered, and determine to what extent the reduction of the chaperones influences the severity of associated neurodegenerative diseases.

\section{REFERENCES}

Arnold, C., Reisch, N., Leibold, C., Becker, S., Prufert, K., Sautter, K., et al. (2004). Structure-function analysis of the cysteine string protein in Drosophila: cysteine string, linker and C terminus. J. Exp. Biol. 207, 1323-1334. doi: $10.1242 /$ jeb.00898

Braun, J. E., Wilbanks, S. M., and Scheller, R. H. (1996). The cysteine string secretory vesicle protein activates Hsc70 ATPase. J. Biol. Chem. 271, 25989-25993. doi: 10.1074/jbc.271.42.25989

Burgoyne, R. D., and Morgan, A. (2015). Cysteine string protein (CSP) and its role in preventing neurodegeneration. Semin. Cell Dev. Biol. 40, 153-159. doi: 10.1016/j.semcdb.2015.03.008

Chamberlain, L. H., and Burgoyne, R. D. (2000). Cysteine-string protein: the chaperone at the synapse. J. Neurochem. 74, 1781-1789. doi: 10.1046/j.1471-4159.2000.0741781.x

Chandra, S., Gallardo, G., Fernandez-Chacon, R., Schluter, O. M., and Sudhof, T. C. (2005). $\alpha$-synuclein cooperates with CSP $\alpha$ in preventing neurodegeneration. Cell 123, 383-396. doi: 10.1016/j.cell.2005.09.028

Donnelier, J., and Braun, J. E. (2014). CSP $\alpha$-chaperoning presynaptic proteins. Front. Cell. Neurosci. 8:116. doi: 10.3389/fncel.2014.00116

Fernández-Chacon, R., Wolfel, M., Nishimune, H., Tabares, L., Schmitz, F., Castellano-Munoz, M., et al. (2004). The synaptic vesicle protein $\operatorname{CSP} \alpha$ prevents presynaptic degeneration. Neuron 42, 237-251. doi: 10.1016/S0896-6273(04)00190-4

García-Junco-Clemente, P., Cantero, G., Gomez-Sanchez, L., Linares-Clemente, P., Martinez-Lopez, J. A., Lujan, R., et al. (2010). Cysteine string protein- $\alpha$ prevents activity-dependent degeneration in GABAergic synapses. J. Neurosci. 30, 7377-7391. doi: 10.1523/JNEUROSCI.0924-10.2010

Greaves, J., Lemonidis, K., Gorleku, O. A., Cruchaga, C., Grefen, C., and Chamberlain, L. H. (2012). Palmitoylation-induced aggregation of cysteinestring protein mutants that cause neuronal ceroid lipofuscinosis. J. Biol. Chem. 287, 37330-37339. doi: 10.1074/jbc.M112.389098

Henderson, M. X., Wirak, G. S., Zhang, Y. Q., Dai, F., Ginsberg, S. D., Dolzhanskaya, N., et al. (2016). Neuronal ceroid lipofuscinosis with DNAJC5/CSP $\alpha$ mutation has PPT1 pathology and exhibit aberrant protein palmitoylation. Acta Neuropathol. 131, 621-637. doi: $10.1007 / \mathrm{s} 00401-015-1512-2$

Hennessy, F., Nicoll, W. S., Zimmermann, R., Cheetham, M. E., and Blatch, G. L. (2005). Not all J domains are created equal: implications for the specificity of Hsp40-Hsp70 interactions. Prot. Sci. 14, 1697-1709. doi: 10.1110/ps.0514 06805

Kashyap, S. S., Johnson, J. R., McCue, H. V., Chen, X., Edmonds, M. J., Ayala, M., et al. (2014). Caenorhabditis elegans dnj-14, the orthologue of the DNAJC5 gene mutated in adult onset neuronal ceroid lipofuscinosis, provides a new platform for neuroprotective drug screening and identifies a SIR-2.1-independent

\section{AUTHOR CONTRIBUTIONS}

Experiments shown in Figure 2 were performed by EL. EL, RR, and LT conceived and wrote the manuscript.

\section{FUNDING}

This work was supported by grants from the Spanish Ministry of Science and Innovation (BFU2013-43763-P) and the Tatiana Perez de Guzman Foundation to LT. RR was supported by a contract from the $V$ Plan Propio of the University of Seville.

\section{ACKNOWLEDGMENTS}

We are grateful to Rafael Fernández-Chacón for discussions and comments on the manuscript.

action of resveratrol. Hum. Mol. Genet. 23, 5916-5927. doi: 10.1093/hmg/ ddu316

Nosková, L., Stranecky, V., Hartmannova, H., Pristoupilova, A., Baresova, V., Ivanek, R., et al. (2011). Mutations in DNAJC5, encoding cysteinestring protein alpha, cause autosomal-dominant adult-onset neuronal ceroid lipofuscinosis. Am. J. Hum. Genet. 89, 241-252. doi: 10.1016/j.ajhg.2011. 07.003

Rozas, J. L., Gomez-Sanchez, L., Mircheski, J., Linares-Clemente, P., NietoGonzalez, J. L., Vazquez, M. E., et al. (2012). Motorneurons require cysteine string protein- $\alpha$ to maintain the readily releasable vesicular pool and synaptic vesicle recycling. Neuron 74, 151-165. doi: 10.1016/j.neuron.2012.02.019

Ruiz, R., Biea, I. A., and Tabares, L. (2014). $\alpha$-Synuclein A30P decreases neurodegeneration and increases synaptic vesicle release probability in CSP $\alpha$-null mice. Neuropharmacology 76(Pt A), 106-117. doi: 10.1016/j.neuropharm.2013.08.032

Ruiz, R., Casanas, J. J., Sudhof, T. C., and Tabares, L. (2008). Cysteine string protein- $\alpha$ is essential for the high calcium sensitivity of exocytosis in a vertebrate synapse. Eur. J. Neurosci. 27, 3118-3131. doi: 10.1111/j.1460-9568.2008.06301.x

Ruiz, R., Lin, J., Forgie, A., Foletti, D., Shelton, D., Rosenthal, A., et al. (2005). Treatment with trkC agonist antibodies delays disease progression in neuromuscular degeneration (NMD) mice. Hum. Mol. Genet. 14, 1825-1837. doi: 10.1093/hmg/ddi189

Schmitz, F., Tabares, L., Khimich, D., Strenzke, N., de la Villa-Polo, P., Castellano-Munoz, M., et al. (2006). CSP $\alpha$-deficiency causes massive and rapid photoreceptor degeneration. Proc. Natl. Acad. Sci. U.S.A. 103, 2926-2931. doi: 10.1073/pnas.0510060103

Sharma, M., Burre, J., Bronk, P., Zhang, Y., Xu, W., and Sudhof, T. C. (2012a). $\mathrm{CSP} \alpha$ knockout causes neurodegeneration by impairing SNAP-25 function. ЕМBO J. 31, 829-841. doi: 10.1038/emboj.2011.467

Sharma, M., Burre, J., and Sudhof, T. C. (2011). CSP $\alpha$ promotes SNARE-complex assembly by chaperoning SNAP-25 during synaptic activity. Nat. Cell Biol. 13, 30-39. doi: $10.1038 /$ ncb2131

Sharma, M., Burre, J., and Sudhof, T. C. (2012b). Proteasome inhibition alleviates SNARE-dependent neurodegeneration. Sci. Transl. Med. 4:147ra113. doi: 10.1126/scitranslmed.3004028

Washbourne, P., Thompson, P. M., Carta, M., Costa, E. T., Mathews, J. R., Lopez-Bendito, G., et al. (2002). Genetic ablation of the t-SNARE SNAP25 distinguishes mechanisms of neuroexocytosis. Nat. Neurosci. 5, 19-26. doi: $10.1038 / \mathrm{nn} 783$

Zhang, Y. Q., and Chandra, S. S. (2014). Oligomerization of Cysteine String Protein alpha mutants causing adult neuronal ceroid lipofuscinosis. Biochim. Biophys. Acta 1842, 2136-2146. doi: 10.1016/j.bbadis.2014.07.009

Zhang, Y. Q., Henderson, M. X., Colangelo, C. M., Ginsberg, S. D., Bruce, C., $\mathrm{Wu}, \mathrm{T}$., et al. (2012). Identification of $\operatorname{CSP} \alpha$ clients reveals a role 
in dynamin 1 regulation. Neuron 74, 136-150. doi: 10.1016/j.neuron.2012. 01.029

Zinsmaier, K. E. (2010). Cysteine-string protein's neuroprotective role. J. Neurogenet. 24, 120-132. doi: 10.3109/01677063.2010.489625

Zinsmaier, K. E., Eberle, K. K., Buchner, E., Walter, N., and Benzer, S. (1994). Paralysis and early death in cysteine string protein mutants of Drosophila. Science 263, 977-980. doi: 10.1126/science.8310297

Zinsmaier, K. E., Hofbauer, A., Heimbeck, G., Pflugfelder, G. O., Buchner, S., and Buchner, E. (1990). A cysteine-string protein is expressed in retina and brain of Drosophila. J. Neurogenet. 7, 15-29. doi: 10.3109/01677069009084150
Conflict of Interest Statement: The authors declare that the research was conducted in the absence of any commercial or financial relationships that could be construed as a potential conflict of interest.

Copyright (C) 2017 Lopez-Ortega, Ruiz and Tabares. This is an open-access article distributed under the terms of the Creative Commons Attribution License (CC BY). The use, distribution or reproduction in other forums is permitted, provided the original author(s) or licensor are credited and that the original publication in this journal is cited, in accordance with accepted academic practice. No use, distribution or reproduction is permitted which does not comply with these terms. 